\title{
Focused Ion Beam Preparation of All Solid State Nanobattery for TEM In-situ Cycling Studies
}

\author{
L. Dupont*, A. Brazier*, D. McGrouther**, N. Hondow***, C. Davoisne*, R. Brydson*** \\ * Laboratoire de Réactivité et de Chimie des Solides, Université de Picardie Jules Verne et UMR \\ 6007; ALISTORE-ERI, 33 rue Saint Leu, 80039 Amiens cedex, France \\ ** Kelvin Nanocharacterisation Centre, Department of Physics\& Astronomy, University of \\ Glasgow, Glasgow, G12 8QQ, UK \\ *** Institute for Materials Research \& SuperSTEM, SPEME, University of Leeds, Leeds LS2 9JT, \\ U.K.
}

Batteries performances depend on many factors amongst which the most critical are the selection/synthesis of the appropriate electrode materials and the control of the electrode/electrolyte interfaces upon cycling. In order to address these issues, electrochemists have to design new electrode materials. More importantly they have to 'enter the private life' of a battery through in-situ techniques so as to spot the slightest textural, chemical or structural modification of the electrodes and interfaces. Electron Microscopy is a powerful tool to help scientists throughout these different steps.

Electron Microscopy techniques have been developed, through the ALISTORE-ERI Electron Microscopy platform (AEMP), to allow the observation of Li-ion air sensitive electrode materials. In the same time, from 2000, we are modifying electron microscopes to be able to perform live in-situ observations of Lithium or Lithium ion batteries cycling in the microscopes. We already succeeded in doing in-situ Scanning Electron Microscopy (SEM) observations on polymer [1], solid, or liquid electrolyte lithium ion batteries in an environmental SEM.

The main remaining dream for scientists working on lithium-ion batteries is to be able to realize such in-situ observation, at the atomic scale, in the TEM. There are several tricky technological problems that stand in the way. It is for instance mandatory to have very thin (less than $100 \mathrm{~nm}$ ) and very small samples. Furthermore, the vacuum required to operate the microscope bans the use of a classical liquid or polymer battery technologies. Several teams in the world have selected different approaches to try to bypass the size and vacuum problems such as the use of ionic liquid as electrolyte [2,3] or the building of a solid state battery specially designed for TEM study [4]. Our approach is somehow different: we want/try to succeed in cycling in-situ a "real" lithium-ion battery. In this purpose, we have decided to work with all solid state microbatteries (homemade microbatteries in the early time then commercial ones). Such all solid state microbatteries (figure 1) are bound to overrun the integrated circuits (miniaturized or not) such as smartphone, mainboards, RFiD tags, MEMS. First, using Focused Ion Beam preparation technique, we sliced a full a "nanobattery" (figure2) in order to develop, from an applied point of view, an unique tool for the study of the all solid state microbattery reliability[5]. Then, we are actually working on the modification of the Nanobattery design (Figure 2) to record not only chemical, textural or structural changes during the cycling but also an electric signal showing that the cycling rate is acceptable.

\section{References}

[1] F. Orsini, L. Dupont, B. Beaudoin, S. Grugeon and J.M. Tarascon, International Journal of Inorganic Materials 2, (2000) 701.

[2] Baer et al., J. Mater. Res. 25( 8), (2010) 1541. 
[3] Jian Yu Huang, et al., Science 330, (2010) 1515.

[4] Ogumi et al., Angew. Chem. Int. Ed. 49, (2010) 4414

[5] A. Brazier, L. Dupont, L. Dantras-Laffont, N. Kuwata, J. Kawamura and J-M. Tarascon, Chem. Mat. 20(6), (2008) 2352.

[6] We gratefully acknowledge the Region of Picardie, The French National Research Agency, ALISTORE-ERI and the European Social Fund for financial support.
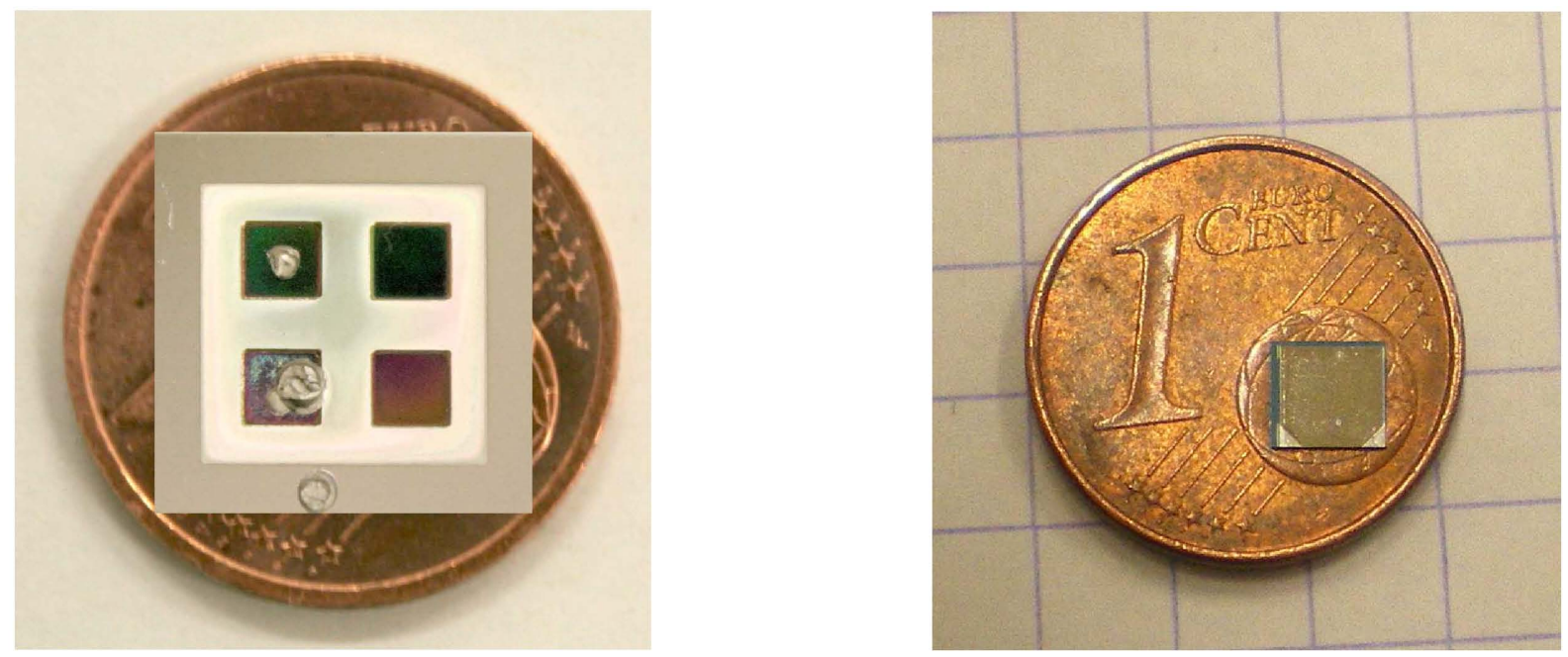

FIG. 1. Pictures of 4 home-made (left) and one commercial (right) microbatteries
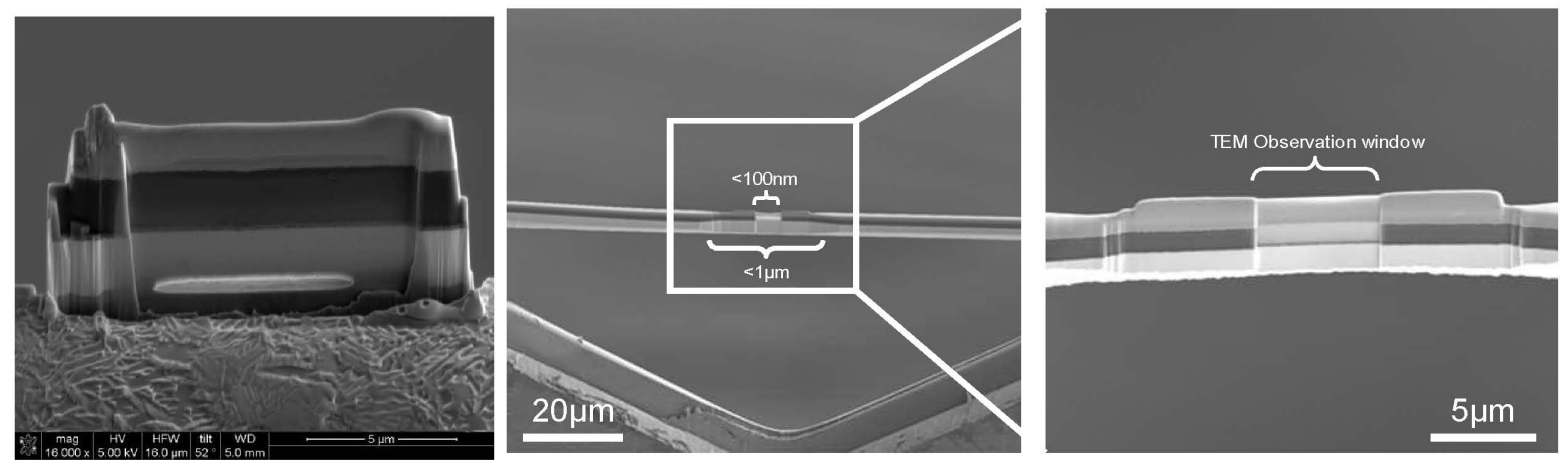

FIG. 2. Picture of the nanobattery prepared by FIB for post-mortem analysis (left) and modified nanobattery design for in-situ measurement (middle and right). 